Korean J. Math. 20 (2012), No. 4, pp. 395-402

http://dx.doi.org/10.11568/kjm.2012.20.4.395

\title{
DIAMETER OF THE DIRECT PRODUCT OF WIELANDT GRAPH
}

\author{
Sooyeon Kim And Byung Chul Song*
}

\begin{abstract}
A digraph $D$ is primitive if there is a positive integer $k$ such that there is a walk of length $k$ between arbitrary two vertices of $D$. The exponent of a primitive digraph is the least such $k$. Wielandt graph $W_{n}$ of order $n$ is known as the digraph whose exponent is $n^{2}-2 n+2$, which is the maximum of all the exponents of the primitive digraphs of order $n$. It is known that the diameter of the multiple direct product of a digraph $W_{n}$ strictly increases according to the multiplicity of the product. And it stops when it attains to the exponent of $W_{n}$. In this paper, we find the diameter of the direct product of Wielandt graphs.
\end{abstract}

\section{Introduction}

A digraph $D=(V, A)$ is primitive if there is a positive integer $k$ such that for each pair $u, v$ of vertices of $D$, there is a directed walk from $u$ to $v$ of length $k$ in $D$. We say that the smallest such $k$ to be the exponent of $D$ and denote it by $\exp (D)$. For each pair of vertices $u, v$ of $D$ if there is a directed walk from $u$ to $v$ of length $k$, then we use the notation $u \stackrel{k}{\longrightarrow} v$. In [7], Wielandt stated that the maximum exponent of the primitive digraphs of order $n$ is $n^{2}-2 n+2$. And he also provide the

Received August 1, 2012. Revised October 5, 2012. Accepted October 10, 2012.

2010 Mathematics Subject Classification: 05C12, 05C20, 05C50.

Key words and phrases: diameter, direct product, Wielandt graph.

This work was supported by the Research Institute of Natural Science of Gangneung-Wonju National University.

*Corresponding author.

(C) The Kangwon-Kyungki Mathematical Society, 2012.

This is an Open Access article distributed under the terms of the Creative commons Attribution Non-Conercial License (http://creativecommons.org/licenses/bync/3.0/) which permits unrestricted non-commercial use, distribution and reproduction in any medium, provided the original work is properly cited. 
digraph, say the Wielandt graph $W_{n}$, which has the maximum exponent for all primitive digraph of order $n$. See also [5]. The Wielandt graph $W_{n}=(V, A)$ is a digraph with the vertex set $V=\{0,1,2, \ldots, n-1\}$ and the arc set $A=\{(i, i+1) \mid 0 \leq i \leq n-2\} \bigcup\{(n-1,0),(n-1,1)\}$. For example, Wielandt graph of order 5 is as in Figure 1.

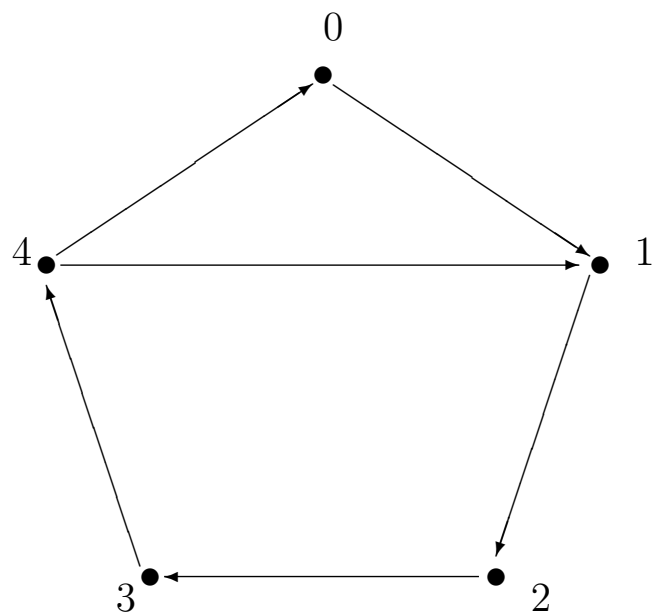

Figure 1. Wielandt graph $W_{5}$ of order 5

For a digraph $D=(V, A)$, the distance $\operatorname{dist}(u, v)$ from a vertex $u$ to a vertex $v$ is the smallest $k$ such that $u \stackrel{k}{\longrightarrow} v$.

The diameter of the digraph $D$ is defined by

$$
\operatorname{diam}(D)=\max _{u, v \in V}\{\operatorname{dist}(u, v)\} .
$$

It is obvious that $D$ is strongly connected if and only if $\operatorname{diam}(D)$ is finite. Moon [4] found a relation between the diameter, the minimum degree and the number of vertices of a graph. The relation implies that if a graph with $n$ vertices has diameter $d \geq 3$ and has minimum degree $r \geq 2$, then $d \leq \frac{3 n-2 r-6}{r}$.

For two digraphs $D=\left(V_{D}, A_{D}\right), E=\left(V_{E}, A_{E}\right)$, define the direct product $D \times E=(V, A)$ of $D$ and $E$ by a digraph where

$$
V=V_{D} \times V_{E}
$$

and

$$
A=\left\{\left(\left(u_{1}, u_{2}\right),\left(v_{1}, v_{2}\right)\right) \mid\left(u_{1}, v_{1}\right) \in A_{D} \text { and }\left(u_{2}, v_{2}\right) \in A_{E}\right\} .
$$


Weichsel [6] and MacAndrew [3] studied the connectivity of the direct product of graphs or digraphs. Lamprey and Barnes [2] showed that the exponent of the direct product of two digraphs $D$ and $E$ satisfies

$$
\exp (D \times E)=\max \{\exp (D), \exp (E)\} .
$$

As an example of the direct product of digraphs, we consider the direct product of Wielandt graph of order 5 . $W_{5} \times W_{5}$ is the digraph with vertex set $\{(i, j) \mid 0 \leq i, j \leq 4\}$ and $\left((i, j),\left(i^{\prime}, j^{\prime}\right)\right)$ is an arc only when (1) $i^{\prime}=i+1$ and $j^{\prime}=j+1$ for $0 \leq i, j \leq 3$, (2) $i^{\prime}=0$ or 1 with $i=4$ and $j^{\prime}=j+1$ for $0 \leq j \leq 3$, (3) $j^{\prime}=0$ or 1 with $j=4$, and $i^{\prime}=i+1$ for $0 \leq i \leq 3$ or $(4)(i, j)=(4,4)$ and $\left(i^{\prime}, j^{\prime}\right)$ is $(0,0),(0,1),(1,0)$ or $(1,1)$.

Kim, Song and Hwang [1] showed that the diameter of the multiple direct product of a primitive digraph $D$ strictly increases and it stops when it attains to the exponent of $D$.

In this paper, we show that the diameter of the direct product of Wielandt graph $W_{n}$ of order $n$ is $\frac{n^{2}}{2}$ for even $n$, and $\frac{n^{2}+1}{2}$ for odd $n$.

\section{Main theorem}

Let $W_{n}$ be the Wielandt graph of order $n$. Note that $\operatorname{diam}\left(W_{n}\right)=$ $\operatorname{dist}(1,0)=n-1$.

For a Wielandt graph of order $n$, the following are strightforward.

1. For $i \neq 0, i \stackrel{k}{\longrightarrow} i$ if and only if $k=p n+q(n-1)$ for nonnegative $p, q$;

2. $0 \stackrel{k}{\longrightarrow} 0$ if and only if $k=p n+q(n-1)$ for positive $p$ and nonnegative $q$.

From now on we use $\alpha_{(n)}$ if there is an integer $p$ such that $\alpha-p n=\alpha_{(n)}$ with $0 \leq \alpha_{(n)} \leq n-1$.

Lemma 1. Let $W_{n}$ be the Wielandt graph of order $n$ and $i$ be vertex of $W_{n}$. If $0 \stackrel{k}{\longrightarrow} i$ for some $k$, then for vertex $d, d \stackrel{k}{\longrightarrow}(d+i)_{(n)}$.

Proof. If $k<n$, then trivially $k=i$ and $d \stackrel{k}{\longrightarrow}(d+i)_{(n)}$.

Let $k \geq n$. In this case since $d \stackrel{p n+q(n-1)}{\longrightarrow} d$ for some nonnegative $p$ and $q$ and $k \geq n>i, k=p n+q(n-1)+i$ where at least one of $p$ and $q$ is positive. If $p \geq 1$, then since

$$
d \stackrel{(n-1)-d}{\longrightarrow}(n-1) \stackrel{2}{\longrightarrow} 1 \stackrel{(p-1) n+q(n-1)}{\longrightarrow} 1 \stackrel{d+i-1}{\longrightarrow}(d+i)_{(n)},
$$


we have $d \stackrel{k}{\longrightarrow}(d+i)_{(n)}$. If $q \geq 1$, then since

$$
d \stackrel{(n-1)-d}{\longrightarrow}(n-1) \stackrel{1}{\longrightarrow} 1 \stackrel{p n+(q-1)(n-1)}{\longrightarrow} 1 \stackrel{d+i-1}{\longrightarrow}(d+i)_{(n)},
$$

we have $d \stackrel{k}{\longrightarrow}(d+i)_{(n)}$.

Note that the converse of Lemma 1 doesn't hold. For example, $n-$ $1 \stackrel{2}{\longrightarrow} 2=((n-1)+3)_{(n)}$, but $0 \stackrel{2}{\longrightarrow} 3$ is impossible.

Lemma 2. The diameter of the direct product $W_{n} \times W_{n}$ of Wielandt graphs satisfies

$$
\operatorname{diam}\left(W_{n} \times W_{n}\right)=\max _{0 \leq i, j \leq n-1} \operatorname{dist}((0,0),(i, j)) .
$$

Proof. By the definition

$$
\operatorname{diam}\left(W_{n} \times W_{n}\right) \geq \max _{0 \leq i, j \leq n-1} \operatorname{dist}((0,0),(i, j)) .
$$

If $\operatorname{dist}\left((\alpha, \beta),\left((\alpha+i)_{(n)},(\beta+j)_{(n)}\right)\right)=k$ and $l \leq k-1$, then at least one of $\alpha \stackrel{l}{\longrightarrow}(\alpha+i)_{(n)}$ and $\beta \stackrel{l}{\longrightarrow}(\beta+j)_{(n)}$ is impossible. By Lemma 1 , at least one of $0 \stackrel{l}{\longrightarrow} i$ and $0 \stackrel{l}{\longrightarrow} j$ is impossible. Therefore $\operatorname{dist}((0,0),(i, j)) \geq k$ and we get

$$
\begin{aligned}
\operatorname{diam}\left(W_{n} \times W_{n}\right) & =\max _{0 \leq \alpha, \beta, i, j \leq n-1} \operatorname{dist}\left((\alpha, \beta),\left((\alpha+i)_{(n)},(\beta+j)_{(n)}\right)\right) \\
& \leq \max _{0 \leq i, j \leq n-1} \operatorname{dist}((0,0),(i, j)) .
\end{aligned}
$$

Proposition 1. For $n$ is even, the diameter of the direct product $W_{n} \times W_{n}$ of Wielandt graphs of order $n$ is $\frac{n^{2}}{2}$.

Proof. Since $(0,0) \stackrel{i}{\longrightarrow}(i, i)$ and $i \leq n-1$, we have

$$
\operatorname{dist}((0,0),(i, i))=i \leq n-1<\frac{n^{2}}{2} \text {. }
$$

Since $\operatorname{dist}((0,0),(i, j))=\operatorname{dist}((0,0),(j, i))$, there is no loss of generality we may assume that $0 \leq i<j \leq n-1$.

If $j-i<\frac{n}{2}$, then $j-i \leq \frac{n-2}{2}$. Since $0 \stackrel{i}{\longrightarrow} i \stackrel{(j-i) n}{\longrightarrow} i$ and $0 \stackrel{j}{\longrightarrow}$ $j \stackrel{(j-i)(n-1)}{\longrightarrow} j$, there is a walk from $(0,0)$ to $(i, j)$ of length $i+(j-i) n$. We have

$$
i+(j-i) n \leq i+\frac{n-2}{2} n=(n+1)+\frac{n-2}{2} n<\frac{n^{2}}{2} .
$$


If $j-i \geq \frac{n}{2}$, then $n-(j-i) \leq \frac{n}{2}$ and $i \leq j-\frac{n}{2} \leq \frac{n-2}{2}$. If we assume that $i \neq 0$, then $0 \stackrel{i}{\longrightarrow} i \stackrel{(n-(j-i))(n-1)}{\longrightarrow} i$ and $0 \stackrel{j}{\longrightarrow} j \stackrel{n(n-(j-i)-1)}{\longrightarrow} j$. Hence there is a walk from $(0,0)$ to $(i, j)$ of length $i+(n-(j-i))(n-1)$. We have

$$
i+(n-(j-i))(n-1) \leq i+\frac{n}{2}(n-1)=\frac{n-2}{2}+\frac{n}{2}(n-1) \leq \frac{n^{2}}{2} .
$$

If $i=0$ and $j=\frac{n}{2}$, then $0 \stackrel{\left(\frac{n}{2}\right) n}{\longrightarrow} 0$ and $0 \stackrel{\frac{n}{2}}{\longrightarrow} \frac{n}{2} \stackrel{\frac{n}{2}(n-1)}{\longrightarrow} \frac{n}{2}$. There is a walk from $(0,0)$ to $(i, j)$ of length $\frac{n^{2}}{2}$.

If $i=0$ and $j>\frac{n}{2}$, then $n-j<\frac{n}{2}$ and so $n-j \leq \frac{n-2}{2}$. Since $0 \stackrel{n+(n-j)(n-1)}{\longrightarrow} 0$ and $0 \stackrel{j+(n-j) n}{\longrightarrow} j$, we have $(0,0) \stackrel{j+(n-j) n}{\longrightarrow}(0, j)$. In this case we have

$$
j+n(n-j) \leq n-1+n\left(\frac{n-2}{2}\right)=\frac{n^{2}-2}{2}<\frac{n^{2}}{2} .
$$

Therefore for $0 \leq i, j \leq n-1$, we have $\operatorname{dist}[(0,0),(i, j)] \leq \frac{n^{2}}{2}$. Moreover we have

$$
\operatorname{diam}\left(W_{n} \times W_{n}\right) \geq \max _{0 \leq i, j \leq n-1} \operatorname{dist}((0,0),(i, j)) \leq \frac{n^{2}}{2} .
$$

To prove the converse, assume that $(0,0) \stackrel{\alpha}{\longrightarrow}\left(0, \frac{n}{2}\right)$ for some $\alpha \leq$ $\exp \left(W_{n}\right)=n^{2}-2 n+2$. Then we have $\alpha=n+p_{1} n+p_{2}(n-1)=$ $\frac{n}{2}+q_{1} n+q_{2}(n-1)$ for some nonnegative integers $p_{1}, p_{2}, q_{1}, q_{2}$ and with $0 \leq p_{2}, q-2 \leq n-1$. It is enough to show that $\alpha \geq \frac{n^{2}}{2}$. By (1), we have

$$
n\left[1+2\left(p_{1}-q_{1}\right)-2\left(q_{2}-p_{2}\right)\right]=2\left(q_{2}-p_{2}\right) .
$$

Since $2-2 n \leq 2\left(p_{2}-q_{2}\right) \leq 2 n-2$ and $1+2\left(p_{1}-q_{1}\right)-2\left(q_{2}-p_{2}\right) \neq 0$, we know that $2\left(p_{2}-q_{2}\right)$ is $n$ or $-n$. If $p_{2}=q_{2}+\frac{n}{2}$, then $p_{2} \geq \frac{n}{2}$. We have

$$
\alpha=n+p_{1} n+p_{2}(n-1) \geq n+\frac{n}{2}(n-1)=\frac{n^{2}+n}{2}>\frac{n^{2}}{2} .
$$

If $q_{2}=p_{2}+\frac{n}{2}$, then $q_{2} \geq \frac{n}{2}$. As a consequence

$$
\alpha=\frac{n}{2}+q_{1} n+q_{2}(n-1) \geq \frac{n}{2}+\frac{n}{2}(n-1)=\frac{n^{2}}{2} .
$$

Proposition 2. For $n$ is odd, the diameter of the direct product $W_{n} \times W_{n}$ of Wielandt graphs of order $n$ is $\frac{n^{2}+1}{2}$. 
Proof. If $j-i<\frac{n}{2}$, then $j-i \leq \frac{n-1}{2}$. Since $0 \stackrel{i}{\longrightarrow} i \stackrel{(j-i) n}{\longrightarrow} i$ and $0 \stackrel{j}{\longrightarrow} j \stackrel{(j-i)(n-1)}{\longrightarrow} j$, there is a walk from $(0,0)$ to $(i, j)$ of length $i+(j-i) n$. We have

$$
\begin{aligned}
i+(j-i) n & =(j-i)(n-1)+j \leq \frac{(n-1)(n+1)}{2} \\
& \leq \frac{n^{2}-1}{2}<\frac{n^{2}+1}{2} .
\end{aligned}
$$

If $j-i>\frac{n}{2}$, then $n-(j-i)<\frac{n}{2}$ and so $n-(j-i) \leq \frac{n-1}{2}$. Since $j \leq n-1, i \leq(n-1)-(j-i) \leq \frac{n-1}{2}-1 \leq \frac{n-3}{2}$. Let us assume that $i \neq 0$. Since $0 \stackrel{i}{\longrightarrow} i \stackrel{(n-(j-i))(n-1)}{\longrightarrow} i$ and $0 \stackrel{j}{\longrightarrow} j \stackrel{n(n-(j-i)-1)}{\longrightarrow} j$, there is a walk from $(0,0)$ to $(i, j)$ of length $i+(n-(j-i))(n-1)$. We have

$$
\begin{aligned}
i+(n-(j-i))(n-1) & \leq \frac{n-3}{2}+\left(\frac{n-1}{2}\right)(n-1) \\
& =\frac{n^{2}-n-2}{2} \leq \frac{n^{2}+1}{2} .
\end{aligned}
$$

If $i=0$ and $j=\frac{n+1}{2}$, then since

$$
0 \stackrel{1}{\longrightarrow} 1 \stackrel{\left(\frac{n-1}{2}\right)(n-1)}{\longrightarrow} 1 \stackrel{n-1}{\longrightarrow} 0
$$

and

$$
0 \stackrel{\frac{n+1}{2}}{\longrightarrow} \frac{n+1}{2} \stackrel{\left(\frac{n-1}{2}\right) n}{\longrightarrow} \frac{n+1}{2},
$$

there is a walk from $(0,0)$ to $(i, j)$ of length $\frac{n^{2}+1}{2}$.

If $i=0$ and $j>\frac{n+1}{2}$, then $n-j<\frac{n-1}{2}$ and so $n-j \leq \frac{n-3}{2}$. Since $0 \stackrel{n+(n-j)(n-1)}{\longrightarrow} 0$ and $0 \stackrel{j+(n-j) n}{\longrightarrow} j$, we have $(0,0) \stackrel{j+(n-j) n}{\longrightarrow}(0, j)$. In this case we have

$$
\begin{aligned}
j+n(n-j) & \leq n-1+n\left(\frac{n-3}{2}\right) \\
& =\frac{n^{2}-n-2}{2}<\frac{n^{2}+1}{2} .
\end{aligned}
$$

Hence for $0 \leq i, j \leq n-1$ with odd $n$, we conclude that $\operatorname{dist}((0,0),(i, j)) \leq$ $\frac{n^{2}+1}{2}$.

If $(0,0) \stackrel{\alpha}{\longrightarrow}\left(0, \frac{n+1}{2}\right)$, then

$$
\alpha=n+p_{1} n+p_{2}(n-1)=\frac{n+1}{2}+q_{1} n+q_{2}(n-1)
$$


for some nonnegative integers $p_{1}, p_{2}, q_{1}, q_{2}$. It is enough to show that $\alpha \geq \frac{n^{2}+1}{2}$. We have

$$
2 n\left(p_{1}-q_{1}\right)=\left(2 q_{2}-2 p_{2}-1\right)(n-1) .
$$

There are 2 possible cases.

1. $k(n-1)=2\left(p_{1}-q_{1}\right)$ and $2 q_{2}-2 p_{2}-1=k n$, for $k \geq 1$.

2. $k(1-n)=2\left(p_{1}-q_{1}\right)$ and $-\left(2 q_{2}-2 p_{2}-1\right)=k n$, for $k \geq 1$.

If (1) holds, then since

$$
p_{1}=\frac{k(n-1)}{2}+q_{1} \geq \frac{k(n-1)}{2},
$$

we conclude that

$$
\begin{aligned}
\alpha & =n+p_{1} n+p_{2}(n-1) \\
& \geq n+p_{1} n \geq \frac{k(n-1)}{2} n \\
& \geq n+\frac{n-1}{2} n=\frac{n^{2}+n}{2} \geq \frac{n^{2}+1}{2} .
\end{aligned}
$$

If (2) holds, then since

$$
p_{2}=\frac{k(n-1)}{2}+q_{2} \geq \frac{k(n-1)}{2},
$$

we conclude that

$$
\begin{aligned}
\alpha & =n+p_{1} n+p_{2}(n-1) \\
& \geq n+p_{2}(n-1) \geq n+\frac{k(n-1)}{2}(n-1) \\
& \geq n+\frac{n}{2} n-1=\frac{n^{2}+n}{2} \geq \frac{n^{2}+1}{2} .
\end{aligned}
$$

By combining Proposition 1 and 2, we have the following theorem.

Theorem 1. If $W_{n}$ be the Wielandt graph of order $n$, then the diameter $\operatorname{diam}\left(W_{n} \times W_{n}\right)$ of the direct product of $W_{n}$ satisfies

$$
\operatorname{diam}\left(W_{n} \times W_{n}\right)=\left\lfloor\frac{n^{2}+1}{2}\right\rfloor .
$$


In [1], Kim, Song and Hwang showed that the diameter of $W_{n} \times$ $\cdots \times W_{n}$ increases as the multiplicity of the product increases. And it finally stops when it reaches the value $\exp \left(W_{n}\right)=n^{2}-2 n+2$ at which the multiplicity of the product is $n-1$. It is worth computing the diameter of the multiple direct product of the Wielandt graph when the multiplicity varies from 3 to $n-2$.

\section{References}

[1] B. Kim, B. Song and W. Hwang, The exponent of a digraph and the diameter of its multiple direct product, Linear Algebra Appl. 437 (2012), 2601-2612.

[2] R. Lamprey, B. Barnes, Primitivity of products of digraphs, in: Proc. 10th Southeastern Conf. on Combinatorics, Graph Theory and Computing (Florida Atlantic Univ., Boca Raton, Fla., Congress. Numer., XXIII-XXIV 637-644 (1979).

[3] M. McAndrew, On the product of directed graphs, Proc. Amer. Math. Soc. 14 (1963), 101-108.

[4] J. Moon, On the diameter of a graph, Michigan Math. J. 12 (1965), 349-351.

[5] H. Schneider, Wielandt's proof of the exponent inequality for primitive nonnegative matrices, Linear Algebra Appl. 353 (2002), 5-10.

[6] P. Weischel, The Kronecker product of graphs, Proc. Amer. Math. Soc. 12 (1962), 47-52.

[7] H. Wielandt, Unzerleghare, nicht negative Matrizen, Math. Z. 52 (1950), 642-645.

Graduate school of Education

Gangneung-wonju National University

Gangneung 210-702, Korea

E-mail: ksy316@freechal.com

Department of Mathematics

Gangneung-Wonju National University

Gangneung 210-702, Korea

E-mail: bcsong@gwnu.ac.kr 